\title{
Juvenile onset autoinflammatory disease due to a novel mutation in TNFAIP3 (A20)
}

\author{
Shuzo Sato' ${ }^{1}$, Yuya Fujita' ${ }^{1}$ Tomonari Shigemura², Hisanori Matoba², Kazunaga Agematsu², Yuya Sumichika', \\ Makiko Yashiro', Atsushi Ono ${ }^{3}$, Yukihiko Kawasaki ${ }^{2}$, Hiroko Kobayashi', Hiroshi Watanabe ${ }^{1}$, Tomohiro Koga ${ }^{4}$, \\ Atsushi Kawakami ${ }^{4}$ and Kiyoshi Migita ${ }^{{ }^{*}}$
}

\section{Key message}

Novel heterozygous C200A A20/TNFAIP3 gene mutation is responsible for autosomal-dominant juvenile onset autoinflammatory disorder.

\section{Letter}

A20, which is encoded by the TNFAIP3 gene, has been shown to control nuclear factor kappa B (NF-kB) signalling by deubiquitinating receptor-interacting proteins [1]. Recently, heterozygous mutations in the TNFAIP3 gene have been found to cause the haploinsufficiency of A20, which presents as an early-onset autoinflammatory disease [2, 3]. Here, we report a Japanese family containing two cases of autoinflammatory disease which exhibit an identical novel TNFAIP3 mutation.

The patient was a 17-year-old Japanese boy who was referred to our department with a 3-year history of recurrent painful oral ulcer and epigastralgia, accompanied by low-grade fever. He presented with periodic fever and oral aphtha that had occurred for the prior 3 years and was referred to our hospital. After spontaneous resolution of the symptoms, he was regularly followed in our hospital. Three years later, the patient was admitted to our hospital for impaired food intake due to epigastralgia, oral ulcer, and fever. His mother had exhibited oral and genital ulcers and erythema nodosum-like lesions on the upper extremities and had died of septicaemia 2 years prior. His younger sister exhibited similar symptoms from the age of 11 years and was suspected of Behçet's disease. Physical examination of the boy revealed painful oral ulcers. Neither genital ulcer nor uveitis was confirmed, but multiple erosions in his stomach mucosa were observed by endoscopic examination.

\footnotetext{
* Correspondence: migita@fmu.ac.jp

${ }^{1}$ Department of Rheumatology, Fukushima Medical University School of Medicine, 1 Hikarigaoka, Fukushima, Fukushima 960-1295, Japan Full list of author information is available at the end of the article
}

Relevant laboratory data are listed in Additional file 1 (Table S1). There were no abnormalities except elevated levels of C-reactive protein (CRP). Anti-nuclear antibodies were positive with low titres; however, tests for other various autoantibodies revealed no abnormalities. The HLA-B haplotype of the patient was not B51 (B39/ B62). Increased serum inflammatory cytokine levels were confirmed at the time of presentation (Additional file 1: Table S1). Although autoimmune phenomena were not prominent, this patient presented with an autoinflammatory phenotype resembling Behçet's disease. Treatment with prednisolone relieved the clinical symptoms and normalized CRP levels.

Recently, Zhou et al. reported 11 patients from six families with a new dominantly inherited autoinflammatory disease, termed haploinsufficiency of A20 (HA20) [4]. This disease has the following characteristics: periodic fever; oral, ocular, and/or genital ulcers; and elevated levels of many cytokines [4]. Serum pro-inflammatory cytokines, particularly tumour necrosis factor (TNF)- $\alpha$, interleukin (IL)-1 $\beta$, IL-6, and IL-8, were elevated in our patient (Additional file 1: Table S1). Therefore, written informed consent for TNFAIP3 gene analysis was obtained from the patient and his father.

The C596_598 del A mutation, which we confirmed by Sanger sequencing (Fig. 1a), introduces a frameshift substitution of alanine for cysteine at position 200, generating a downstream stop codon (Cys200Alafsx16; C200Afs"16) in the OTU domain of A20. This heterozygous C200A fs*16 mutation was present in the patient's younger sister, who showed similar symptoms. Sanger sequencing of the unaffected father showed no variant of TNFAIP3, suggesting that these mutations were maternally derived. This variant is absent from public databases (ExAs/dbSNP).

To address the molecular basis of this C200Afs"16 mutation, we stimulated peripheral blood mononuclear cells (PBMCs) that were isolated from the patient and

(c) The Author(s). 2018 Open Access This article is distributed under the terms of the Creative Commons Attribution 4.0 International License (http://creativecommons.org/licenses/by/4.0/), which permits unrestricted use, distribution, and 


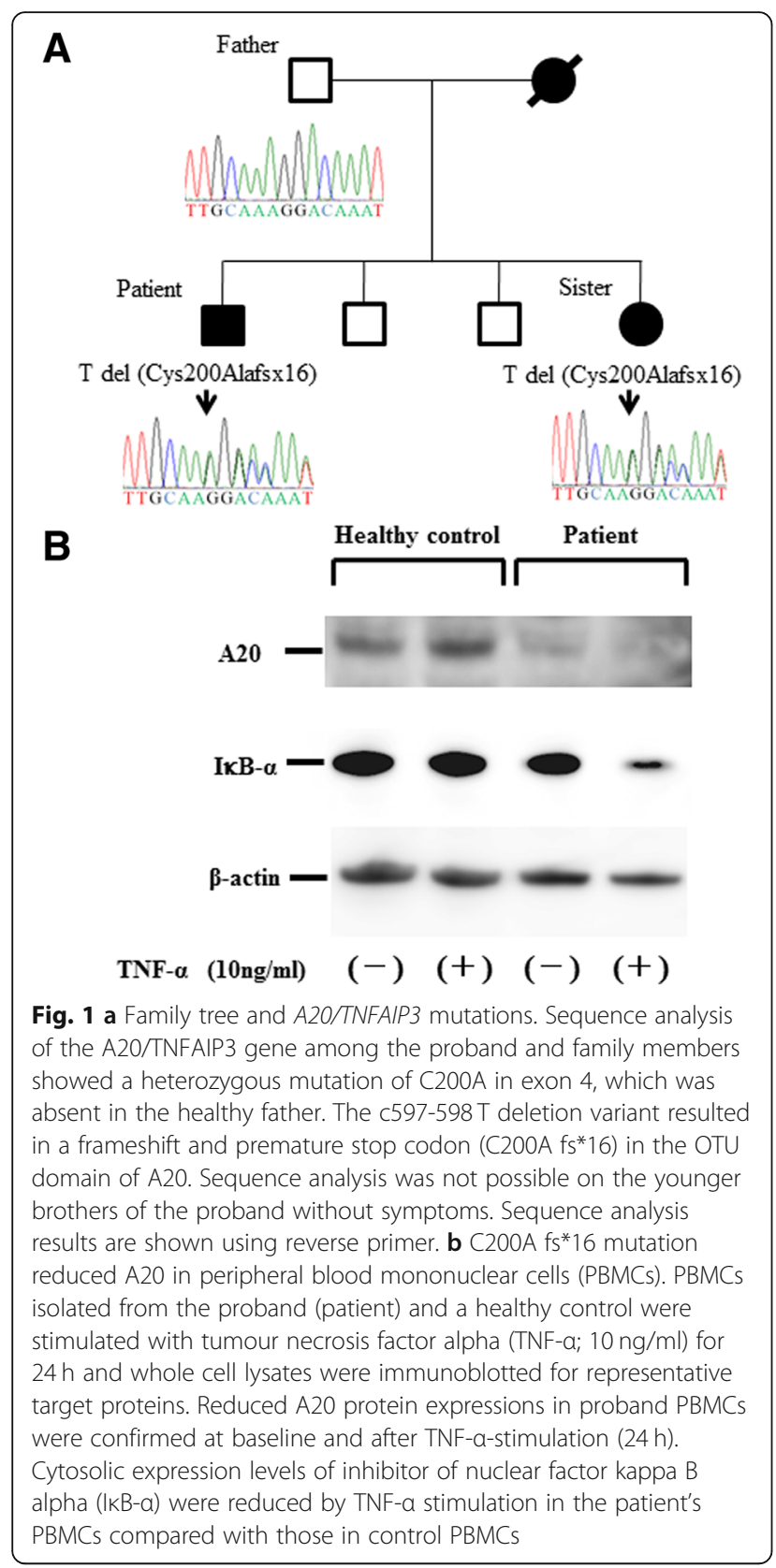

from a healthy subject with TNF- $\alpha(10 \mathrm{ng} / \mathrm{ml})$, and then performed immunoblot blot analysis with anti-A20 antibodies (Fig. 1b). Wild-type A20 protein expression was reduced in patient-derived PBMCs compared with those from the healthy subject at baseline and after TNF- $\alpha$ stimulation. These results suggested that the frameshift mutation identified in the present case resulted in impaired expression of A20 at baseline and after TNF- $\alpha$ stimulation. Additionally, cytosolic inhibitor of nuclear factor kappa B alpha (IкB- $\alpha$ ) protein levels were reduced in patient-derived PBMCs after stimulation with TNF- $\alpha$, whereas IkB- $\alpha$ protein levels were unchanged in control PBMCs. These findings suggest that IKB- $\alpha$ degradation was accelerated by TNF- $\alpha$ stimulation in PBMCs isolated from the patient.

A20 is a ubiquitin-editing enzyme that inhibits key proinflammatory molecules, including inhibitor of nuclear factor kappa B kinase (IKK) $\gamma$ [5]. Zhou et al. reported that unrelated family cases manifested autoinflammation with heterozygous mutations in TNFAIP3; all patients exhibited oral and genital ulcers [4]. The clinical manifestations seen in our patients were consistent with those of the previously reported cases [6], whereas the disease onset (at 14 years old) was relatively late compared with those of the reported cases [6]. We identified a novel heterozygous missense mutation, which was located at the OTU domain of A20 in both affected siblings in this family.

In previously reported HA20 cases, mutant cells have shown enhanced NF- $\mathrm{kB}$ activity, as demonstrated by increased phosphorylation of $I K K \alpha / \beta$ and increased degradation of IkB- $\alpha$ [7]. In the PBMCs isolated from our patient, cellular IкB- $\alpha$ failed to maintain baseline levels compared with cells expressing wild-type A20 in response to extended TNF- $\alpha$ stimulation. IKK $\alpha / \beta$ mediated phosphorylation of IкB- $\alpha$ resulted in IкB- $\alpha$ degradation and subsequent nuclear translocation of NF- $\mathrm{B}$ [8]. These findings suggest that haploinsufficiency of TNFAIP3, which leads to impaired A20 expression, could be responsible for the TNF- $\alpha$-induced increased degradation of IKB- $\alpha$ in the present case. Although the molecular pathogenesis of our patient and previously reported cases may be similar, variations in clinical manifestations suggest additional modifying factors [6]. Further studies regarding functional and genetic analysis of A20 will elucidate the pathogenesis of A20-mediated autoimmune/autoinflammatory diseases.

\section{Additional file}

Additional file 1: Table S1. Laboratory findings and cytokine/chemokine profile on admission. (PDF $131 \mathrm{~kb}$ )

\section{Abbreviations}

HA20: Haploinsufficiency of A20; IKK: Inhibitor of nuclear factor kappa B kinase; IL: Interleukin; IKB-a: Inhibitor of nuclear factor kappa B alpha; PBMC: Peripheral blood mononuclear cell; TNF: Tumour necrosis factor

\section{Acknowledgements}

Not applicable.

\section{Funding}

The study was supported by the Practical Research Project for Rare/ Intractable Diseases from the Japanese Agency for Medical Research and Development (AMED).

Availability of data and materials

Please contact the corresponding author for data requests. 


\section{Authors' contributions}

TS, HM, and KA carried out the molecular genetic studies, participated in the sequence alignment, and drafted the manuscript. YF, YS, MY, TK, and KM carried out the immunoassays. $\mathrm{AO}$ and $\mathrm{YK}$ participated in the sequence alignment. KM and AK participated in the design of the study. SS, HK, and HW conceived of the study, and participated in its design and coordination, and helped to draft the manuscript. All authors read and approved the final manuscript.

\section{Ethics approval and consent to participate}

Ethical approval for this study (no. 2920) was provided by the Ethics Committee of Fukushima Medical University and written informed consent was obtained from each individual.

\section{Consent for publication}

Written informed consents were obtained from all subjects for publication of this case report.

\section{Competing interests}

KM has received research grants from Chugai, Pfizer, and AbbVie. The remaining authors declare that they have no competing interests.

\section{Publisher's Note}

Springer Nature remains neutral with regard to jurisdictional claims in published maps and institutional affiliations.

\section{Author details}

'Department of Rheumatology, Fukushima Medical University School of Medicine, 1 Hikarigaoka, Fukushima, Fukushima 960-1295, Japan.

${ }^{2}$ Department of Infectious Immunology, Shinshu University Graduate School of Medicine, Asahi 3-1-1, Matsumoto, Nagano 390-8621, Japan. ${ }^{3}$ Department of Pediatrics, Fukushima Medical University School of Medicine, 1 Hikarigaoka, Fukushima, Fukushima 960-1295, Japan. ${ }^{4}$ Department of Immunology and Rheumatology, Unit of Translational Medicine, Graduate School of Biomedical Sciences, Nagasaki University, Sakamoto1-7-1, Nagasaki 852-8501, Japan

\section{Published online: 10 December 2018}

\section{References}

1. Ma A, Malynn BA. A20: linking a complex regulator of ubiquitylation to immunity and human disease. Nat Rev Immunol. 2012;12(11):774-85.

2. Aeschlimann FA, Batu ED, Canna SW, Go E, Gül A, Hoffmann P, et al. A20 haploinsufficiency (HA20): clinical phenotypes and disease course of patients with a newly recognised NF-kB-mediated autoinflammatory disease. Ann Rheum Dis. 2018;77(5):728-35.

3. Shigemura T, Kaneko N, Kobayashi N, Kobayashi K, Takeuchi Y, Nakano N, et al. Novel heterozygous C243Y A20/TNFAIP3 gene mutation is responsible for chronic inflammation in autosomal-dominant Behçet's disease. RMD Open. 2016;2(1):e000223.

4. Zhou Q, Wang H, Schwartz DM, Stoffels M, Park YH, Zhang Y, et al. Loss-of function mutations in TNFAIP3 leading to A20 haploinsufficiency cause an early-onset autoinflammatory disease. Nat Genet. 2016;48(1):67-73.

5. Wertz IE, O'Rourke KM, Zhou H, Eby M, Aravind L, Seshagiri S, et al. De-ubiquitination and ubiquitin ligase domains of A20 downregulate NF-kappaB signalling. Nature. 2004;430(7000):694-9.

6. Kadowaki T, Ohnishi H, Kawamoto N, Hori T, Nishimura K, Kobayashi C, et al. Haploinsufficiency of A20 causes autoinflammatory and autoimmune disorders. J Allergy Clin Immunol. 2018;141(4):1485-8.

7. Duncan CJA, Dinnigan E, Theobald R, Grainger A, Skelton AJ, Hussain R, et al. Early-onset autoimmune disease due to a heterozygous loss-of-function mutation in TNFAIP3 (A20). Ann Rheum Dis. 2018;77(5):783-6.

8. Chen ZJ. Ubiquitination in signaling to and activation of IKK. Immunol Rev. 2012;246:95-106. 